\title{
Intragastric Migration of Swedish Adjustible Gastric Band
}

\author{
Andrejs Vanags*,**, Ilze Strumfa*,**, Maris Pavars*, Janis Gardovskis*,** \\ *Pauls Stradins Clinical University Hospital, Riga, Latvia \\ ${ }^{* *}$ Riga Stradins University, Latvia
}

\section{Summary}

Swedish adjustable gastric banding (SAGB) is an operative procedure meant for the treatment of morbid obesity. It is characterised by application of low-pressure, high-volume device upon the stomach. One of the major complications, currently described in $6 \%$ of patients, is the intragastric migration of the adjustable gastric band. The removal of the band is the gold standard in these cases and must be performed either urgently or as a scheduled manipulation according to the symptoms. We present a thoroughly documented case of surgical obesity treatment by SAGB complicated by intragastric band migration as well as our experience in settling the complication.

Key words: morbid obesity, bariatric surgery, Swedish adjustable gastric banding, migration.

\section{AIM OF THE DEMONSTRATION}

Here, we present a well-documented case of intragastric Swedish adjustable gastric band migration along with imaging studies and data of surgery in order to add our experience to the published evidence in the bariatric surgery.

\section{CASE REPORT}

Forty five-years-old female sought medical help because of morbid obesity. She weighted $124 \mathrm{~kg}$ and had body mass index (BMI) greater than $45 \mathrm{~kg} / \mathrm{m}^{2}$. As informed patient consent was received, she was included in the protocol for gastric band placement. The protocol consisted of the obesity evaluation, patient education in order to understand the medical condition and achieve weight loss of at least $10 \%$ and operative treatment after successful accomplishment of the prerequisites. The operative procedure was performed by laparoscopic approach in P. Stradin's Clinical University Hospital. During the following 8 months patient's weight reduced for about $50 \mathrm{~kg}$ and BMI decreased to $29 \mathrm{~kg} / \mathrm{m}^{2}$. However, the entire adjustable silicone gastric band device eroded inside the stomach. The complication was initially diagnosed by contrast X-ray surveillance 9 months after the operation. Tight feeling in the epigastrium and sense of fullness gradually appeared over the next 2 weeks. Gastroscopy was performed then and revealed granulation tissues in the dorsal wall of gastric subcardia and a silicone canule that had passed through the stomach wall and reached the prepyloric part of the stomach (figures 1-2). Patient was prepared for laparoscopic removal of the adjustable gastric band under endoscopic control on the next day. Due to the large size of the silicone gastric band exceeding the size of oesophageal lumen and thus precluding the endoscopic evacuation of the device, the attempt to remove the adjustable gastric band by endoscopic assistance was unsuccessful.
Therefore an approximately $3 \mathrm{~cm}$ long incision in the gastric wall was done during the laparoscopy. The gastric band was removed then by endopouch assistance. The incision in the gastric wall and the fault were closed by separate sutures laparoscopically. There were no intraoperative and postoperative complications. The patients' general condition was good and she was discharged on $7^{\text {th }}$ postoperative day in a good physical and mental condition. The laboratory investigation demonstrated no abnormalities.

Later, due to successful weight loss, a cosmetic abdominal wall defect manifested as a skin fold. Therefore an abdominal plastic operation was performed two months after the band removal. Simultaneously with the plastics, the patient benefited from herniorrhaphy in order to treat a concomitant postoperative hernia after the extirpation of uterus and adnexes performed due to uterine myoma one year prior to bariatric surgery. She was discharged in the $4^{\text {th }}$ postoperative day in a good condition.

At present, 19 SAGB have been performed in P. Stradin's Clinical University Hospital, all of them by laparoscopic approach. We have had 2 major complications, but only one of them was associated with the band migration as described.

\section{DISCUSSION}

The Lap-Band system was first described in 1992 and clinically proved in 1994 in USA. Within the 15 years, laparoscopic adjustable banding was found an effective and safe treatment tool for morbid obesity (Forsell et al., 1999; Chevallier et al., 2004). More than 100.000 laparoscopic procedures have been performed since that (Blanco-Engert et al., 2003). Nowadays laparoscopic adjustable gastric banding is the most frequently applied bariatric procedure in Europe (Weiss et al., 2000). The advantages of laparoscopic gastric banding in contrast 
to the gastric bypass or vertical banded gastroplasty consist in the reversibility of the laparoscopic procedure, minimal damage to the stomach and adjustable degree of restriction in every separate case (Langer et al., 2004). Successful weight reduction depends more on the patient in contrast to the bypass malabsorptive procedure (Zehetner et al., 2004). Short-term outcome of the SAGB, characterised by BMI dynamics, seems to be beneficial (Wong et al., 2005). Experiences in other countries showed that SAGB can only restrict solid food, but is less effective if the patient use liquids or semi-solid food with high glucose content (Zehetner et al., 2004). In our patient, SAGB ensured the weight loss. Thus, in our experience, SAGB is an effective method in bariatric surgery.

The reported rate of complications is low (Fried et al., 2004; Zehetner et al., 2004). One of the described complications is the gastric band migration through the gastric wall (Chevallier et al., 2004). Other major complications like port-associated complications, oesophageal dilatation, and band slippage as well as pouch enlargement were observed in long-term studies (Stroh et al., 2008). The band migration through the stomach wall happens mostly during the following two years after procedure (Weiss et al., 2000, Langer et al., 2004) at the rate $0.5 \%$ - 9\% (Forsell et al., 1999; Mittermair et al., 2002; Nocca et al., 2005). Several hypotheses have been proposed as band overfilling, rapid band filling and local infection caused by gastric wall trauma by the implanted band (Weiss et al., 2000, Weiss et al., 2004). Band migration usually is diagnosed by endoscopy or by contrast roentgenogram (Hainaux et al., 2005).

Although our group of patients is still small, the rate of migration $1 / 19(5.3 \%$; $95 \%$ confidence interval $0.9-24.6 \%$ ) is within the reported range. Routinely we perform follow-up by contrast roentgenogram as the migration can be asymptomatic. This approach is substantiated by published data and proved effective in the presented case.

Partial migration can be treated conservatively (Zehetner et al., 2004) while the total migration should be treated operatively. Migration almost never requires urgent surgery (Mittermair et al., 2002). Usually band removal is performed by laparoscopic approach, but there is another alternative published method of managing the band migration (Weiss et al., 2000; Regusci et al., 2003) as endoscopic band removal after cutting the band by the appropriate devise like scissor, laser etc.

Although complications may occur after bariatric surgery, adequate knowledge can help to plan the follow-up and to choose the best treatment options. Gastric band migration is a rare complication but it can be successfully managed.

In conclusion, intragastric Swedish adjustable band migration is one of the possible complications with rate of $5.3 \%$ in our cohort. However, long-term observation of the expanding group of patients may add new evidence. The laparoscopic approach can be used for bariatric surgery and for management of such complications as band migration if these occur. It allows early mobilisation, short hospital stay, early return to work and low risk of wound complications. Occurrence of the complications as described above generates necessity for individual follow-up as well as for the longterm evaluation of complication rate and the efficacy of SAGB.

\section{Conflict of interest: None}

\section{REFERENCES}

1. Blanco-Engert R, Weiner S, Pomhoff I, Matkowitz R, Weiner RA. Outcome after laparoscopic adjustable gastric banding, using the Lap-Band ${ }^{\circledR}$ and the Heliogast ${ }^{\circledast}$ band: a prospective randomised study // Obesity Surgery, 2003; 13:1 - 4

2. Chevallier JM, Zinzindohoue F, Douard R, Blanche JP, Berta JL, Altman JJ, Cugnenc PH. Complications after laparoscopic adjustable gastric banding for morbid obesity: experience with 1.000 patients over 7 years // Obesity Surgery, 2004; 14:407 - 414

3. Forsell P, Hallerback B, Glise H, Hellers G. Complications following Swedish adjustable gastric banding: a long-term follow-up // Obesity Surgery, 1999; 9:11- 16

4. Fried M, Miller K, Kormanova K. Literature review of comparative studies of complications with Swedish band and Lap-Band ${ }^{\circledR} / /$ Obesity Surgery, 2004; 14:256 - 260

5. Hainaux B, Agneessens E, Rubesova E, Muls V, Gaudissart Q, Moschopoulos C, Cadiere GB. Intragastric band erosion after laparoscopic adjustable gastric banding for morbid obesity: imaging characteristics of an underreported complication // ARJ, 2005; 184:109 - 112

6. Langer FB, Bohdjalian A, Hoda A, Silberhumer G, Felberbauer FX, Rasoul-Rockenschaub S, Zacherl J, Wenzl E, Prager G. Early results of laparoscopic adjustable gastric banding using the new lowpressure soft gastric band // Eur Surg, 2004; 36: $345-349$

7. Mittermair RP, Weiss $\mathrm{H}$, Nehoda $\mathrm{H}$, Aigner $\mathrm{F}$. Uncommon intragastric migration of the Swedish adjustable gastric band // Obesity Surgery, 2002; $12: 372-375$

8. Nocca D, Frering V, Gallix B, de Seguin, des Hons C, Noel P, Pierredon M. A, Millat B, Fabre JM. Migration of adjustable banding from a cohort study of 4.236 patients // Surg Endosc, 2005; 19:947 - 950

9. Regusci L, Groebli Y, Meyer JL, Walder J, Margalith D, Schneider R. Gastroscopic removal of an adjustable gastric band after partial intragastric migration // Obesity Surgery, 2003; 13:281 - 284

10. Stroh C, Hohmann U, Will U, Flade-Kuthe R, Herbig B, Höhne S, Köhler, Pick P, Horbach T, Weiner R, Wolff S, Lippert H, Wolf AM, Schmidt U, Meyer F, Manger $\mathrm{T}$ Experiences of two centers of bariatric surgery in the treatment of intragastral band migration after gastric banding - the importance of the German multicenter observational study 
for quality assurance in obesity surgery // Int J Colorectal Dis, 2008, 23:901 - 908

11. Weiss H, Nehoda H, Labeck B, Peer R, Aigner F. Gastroscopic band removal after intragastric migration of adjustable gastric band: a new minimal invasive technique // Obesity Surgery, 2000; 10: $167-170$

12. Wong SKH, So WY, Yau PYP, Chan AKL, Lee S, Chan PN, Chow FCC, Chung SSC. Laparoscopic adjustable gastric banding for the treatment of morbidity obese patients: early outcome in a Chinese cohort // Hong Kong Med J, 2005; 1 1:20 - 29

13. Zehetner J, Holzinger F, Triaca H, Klaiber Ch. A 6year experience with the Swedish adjustable gastric band // Surg Endosc, 2005; 19:21 - 28

\section{Address:}

Andrejs Vanags,

Hereditary Cancer Institute,

Riga Stradins University,

Dzirciema Street 16, LV 1007,

Riga, Latvia,

e-mail: vanags314@inbox.lv

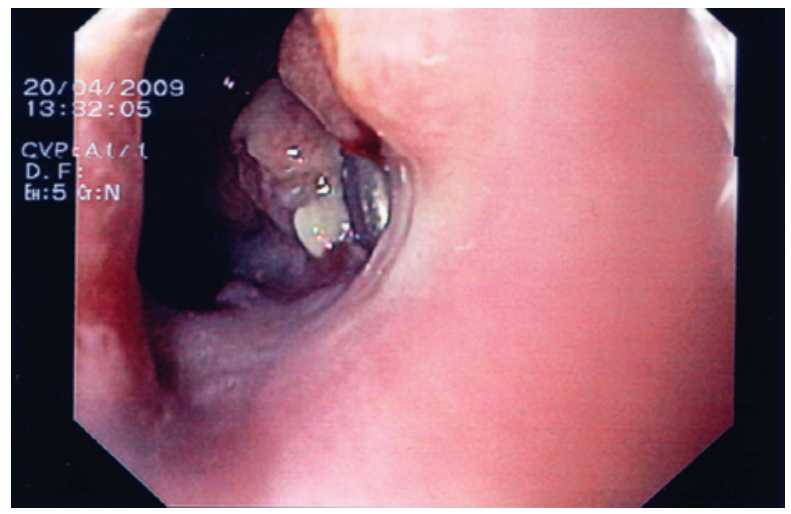

Fig. 1. Adjustable gastric band in the gastric wall

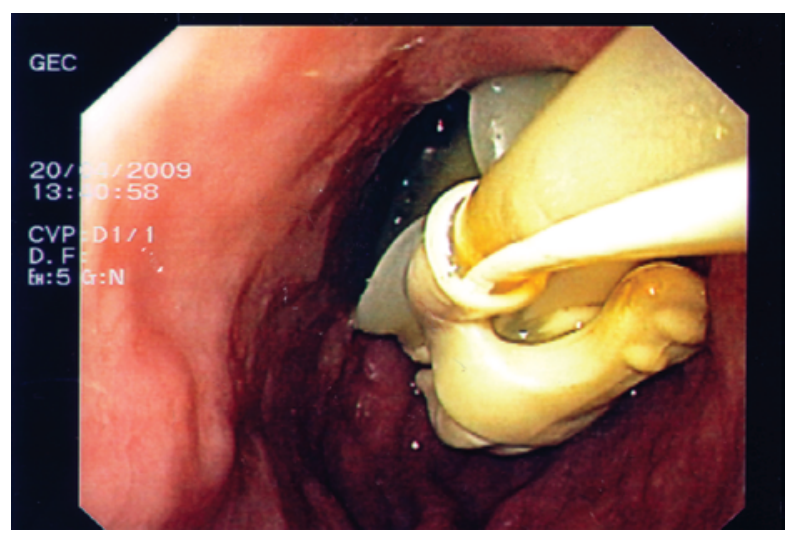

Fig. 2. Silicone adjustable band totally migrated through the stomach wall 